\title{
On the Form and Function of Reflexives in Artificial Languages
}

\author{
Alan Libert and Christo Moskovsky \\ University of Newcastle
}

\begin{abstract}
A bstract
This paper explores issues in relation to the form and function of reflexive pronouns which a language designer should take into consideration in the construction of an artificial language grammar. The first main part of the paper deals with the rather complex and versatile nature of reflexive pronouns in natural languages, discussing reflexives in terms of syntactic and discourse function, morphemic structure, lexical specification for phi-features, subject-orientation, etc. Typological and functional aspects of reflexive pronouns in a number of existing artificial languages are considered in the next part of the paper. The final part addresses the question of what would be the optimal reflexive pronoun from the perspective of artificial language construction. It is proposed that any component of an artificial language grammar, including the reflexive pronoun, would inevitably be a compromise between simplicity of form and function on the one hand, and clarity on the other. It is also suggested that, as artificial languagesarebytheirverynaturenon-primarylanguages, designers of artificial languages should take into account essential properties of second language acquisition and use.
\end{abstract}




\section{Introduction}

Reflexivity is one of the more difficult phenomena of natural languages to analyze.' Even after considerable study, it has proven hard to precisely formulate the conditions under which reflexive pronouns (henceforth RPs) are used, even in English, the most studied language. Possibly partly because of the lack of precise understanding of this area in natural language, it appears that designers of artificial languages (henceforth ALs) usually do not specify how it works in their languages, and perhaps do not give adequate thought to the choice of forms and functions of reflexive words. In this paper, we present a survey of reflexive pronouns in ALs, and then make some suggestions for how this area of the grammar should be constructed. ${ }^{2}$ To our knowledge, little work has been done in this field; Morneau (1994) has proposed guidelines for anaphora in artificial languages, but he does not deal specifically with reflexives. We believe that ALs are often best understood in the context of a comparison to natural languages. Therefore, before discussing the reflexives of ALs, we first describe

\footnotetext{
${ }^{1}$ Weusethefollowing abbreviations in glosses of examples: ABS-absolutive INSTR-instrument(al) ACC-accusative M-masculine ADJ-adjective NOM-nominative ASP-aspect OBJ-object

CAUS-causative PARTIC-participle CL-clitic COMP-complementizer PASS-passive POSS-possessive DETR-detransitivizer PRES-present ERG-ergative REFL-reflexive EXT-extent of action SG-singular FOC-focus VOC-vocative INFIN-infinitive Also,"OT"indicatesthatatranslationisours, ratherthanbeingmadebythesource. Wehave oftennotshown the internal structure ofwordswherethisisnotrelevant

${ }^{2}$ This paperwillnotdeal withlanguages whichhavebeencreatedinconnection with some work of fiction, such as Klingon.
} 
their natural language counterparts. This part of the paper may also prove useful to AL designers, as they can see what possibilities occur in natural languages.

\section{Natural Languages}

Pronouns are a standard feature in natural languages and as such have long been an integral part of linguistic description. One essential property of pronouns is that they have no intrinsic reference of their own and, in order to be interpreted, they need to be linked to a linguistic or discourse entity and derive their reference by way of this link. It is, therefore, hardly surprising that, taxonomic and typological issues apart, there has been a considerable amount of interest in aspects of interpretability of pronouns. The issue received a new dimension after Lees and Klima's (1963) seminal paper 'Rules for English Pronominalization' which related the interpretation of pronouns to their distribution. The paper drew attention to the fact that there is a certain complementarity in the distribution of non-reflexive and reflexive pronouns: informally, reflexives cannot be too far away from, while pronouns cannot be too close to, their antecedents. In the case of English, RPs normally require a clause-internal antecedent:

(1) a. John ${ }_{i}$ despises himself ${ }_{i \cdot}^{3}$

b. ${ }^{*} \mathrm{John}_{i}$ thinks that Mary despises himself ${ }_{i}$

On the other hand, (non-reflexive) pronouns as a rule "look for" an antecedent outside of the clause they are in:

(2) a. John ${ }_{i}$ thinks that Mary despises $\operatorname{him}_{i}$.

\footnotetext{
${ }^{3}$ Assignment of identical indices is a standard way to indicate that two (or more) elements are in a coreferential relation.
} 
100 On the Form and Function of Reflexives in Artificial Languages

b. ${ }^{*} \operatorname{John}_{i}$ despises $\operatorname{him}_{i}{ }^{4}$

Since Lees and Klima's paper, the amount of research devoted to the investigation of anaphora in a range of typologically distinct languages can only be described as colossal and, as a result, knowledge in this area has made remarkable progress. ${ }^{5}$ Studies investigating anaphora can broadly be divided into two main categories: those assuming a structural approach-more often than not within a generative framework, and those assuming a pragmatics-based approach. In the former, the distribution of referentially dependent elements, such as reflexives and pronouns, is subject to the operation of Binding Conditions ( Chomsky 1981, 1986, among many others) which involve a specific configurational relation called c(onstituent)-command (Reinhart 1983) and a locality constraint based on another configurational relation called government ${ }^{7}$; in the pragmatics-based approach, the distribution of reflexives and pronouns is seen as a result of the discourse function they perform, e.g., to disambiguate between two or more potential readings (Dowty 1980), and is analysed in terms of a set of Gricean discourse principles (Levinson 1991). The phenomenon, however, has turned out to be so complex that neither

\footnotetext{
${ }^{4}$ The sentence is ungrammatical only in the indicated reading.

${ }^{5}$ Anaphora isabroadtermforreferentiallydeficient elements; theterm anaphor will occasionally be used in this paper to refer to both reflexive and non-reflexive pronouns.

${ }^{6}$ In an approach assuming that clauses have an internal abstract hierarchical "tree" structure, c-command is a relation between two constituents neither of which dominates theother; the node immediately dominating thec-commanding constituent also dominates the c-commanded constituent. Thus, in a standard clause, the noun phrase which c-commands all other noun phrases is the subject; the significance of this is that, within such a clause, there is no noun phrase that c-commands the subject. Therefore, there is nothing in the clause that qualifies as a potential antecedent for the subject, and it could only take a clause-external antecedent.

${ }^{7}$ This is a relation between a lexical head and another constituent within the same structural category.
} 
structural nor pragmatic approaches have been completely successful in accounting for distributional and/or interpretive aspects of pronouns. There is little doubt that one basic reason for this lack of success is the rather versatile nature of RPs and the contexts they can be used in. In what follows, we discuss properties of reflexive forms in a variety of typologically distinct languages which, in the view taken here, should be taken into account in the construction of an artificial language.

\subsection{Typology of RPs in Natural Languages}

Natural languages employ a variety of devices to mark reflexivity. What we are specifically looking at are RPs or pronoun-like words (possibly, clitics), but it should be noted that reflexivity can be marked with a morphological affix attached to the verb as well. There are also languages which do not have a required distinct reflexive form of either type. For instance, in Gumbaynggir (Eades 1979:312, cited in Levinson 1991:134), the object forms of 3rd person personal pronouns can be interpreted either as coreferential or non-coreferential with the subject:

(3) gula:-du bu:rwang gula:na maga-yu. 3SG-ERG painted 3SG-ABS red paint-INST

"He $e_{i}$ painted him $_{i / j}$ with red paint."

Such languages seem to be extremely rare. Most languages do have RPs and generally form them in the following ways (we give here the classification as in Schladt (1999:105-6)):

(A) from the lexical item 'body', or lexical items for body parts, e.g., "head" (or a form that was diachronically at some stage a lexical item for body part):

\footnotetext{
${ }^{8}$ This is by far the largest group of languages in the corpus.
} 
(4) Kabuverdiano

\begin{tabular}{llll}
\hline Manel & fer? & se & cabe c쨑 \\
Manuel & hurt & 3SG.POSS & head
\end{tabular}

"Manuel hurt himself."

(Estudos lingußBticos crioulos 1967:22, cited in Schladt1999:105)

(B) from elements with meanings such as "person", "owner":

\section{(5) Paumari?}

Abono-ra na-noki-a-a-ha.

self-OBJ CAUS-see-DETR-ASP-THEME

"He sees himself."

(Chapman andDerbyshire1991:178, cited in Schladt 1999:105)

(C) from a lexical item with a meaning of reversal of direction:

(6) $\underline{\text { Sanuma }}$

\begin{tabular}{lllll}
\hline Atakusa & a-nö & kama & nia & sapa \\
gun & 3SG-INSTR & 3SG & shoot & reverse.DIR
\end{tabular}

ko-pa-so-ma.

return-EXT-FOC-COMP

"He shot himself with a gun."

(Borgman 1990:43, cited in Schladt 1999:105)

(D) from a lexical item whose original meaning involved 'reflection':

(7) Finnish

$\begin{array}{lll}\text { Jussi } & \text { näki } & \text { itse-nsä. } \\ \text { Jussi(NOM) } & \text { see.PAST } & \text { reflection.on.water-3SG.POSS }\end{array}$

"He sees himself."

(Faltz 1985:68, cited in Schladt 1999:105) 
(E) from locative prepositions:

(8) $\underline{\text { Zande }}$

$\begin{array}{ll}\text { MRi?m? } & \text { tI?-r } \\ \text { I-kill } & \text { on-me }\end{array}$

"I kill myself."

(Tucker and Bryan 1966:150, cited in Schladt 1999:106)

(F) from items which were originally used as emphatic pronouns, and which may still be used in this way, e.g., the English himself (see section 1.3$)^{9}$.

Whatever their origin, synchronically RPs are universally divided into two general types: simplex forms consisting of a single morpheme (monomorphemic RPs):

(9) Dutch

Oscar gedraagt zich.

"Oscar behaves himself." (Reuland 2001:451)

and complex forms, usually consisting of two morphemes (polymorphemic RPs):

(10) $\underline{\text { Dutch }}$

Oscar haat zichself.

"Oscar hates himself." (Reuland 2001:451)

\footnotetext{
${ }^{9}$ This is apparently a relatively small group, made up exclusively of European languages, according to Schladt (1999).

${ }^{10}$ AwidelyadoptedwaytorefertothesamedistinctionisintermsofSE(=simplex) and SELF (=complex) RPs (see Reinhart and Reuland 1991, 1993).
} 
104 On the Form and Function of Reflexives in Artificial Languages

As the examples above indicate, Dutch has both a simplex and a complex RP, but there are languages (e.g., Russian, Czech) in which there is only a simplex form:

(11) Russian

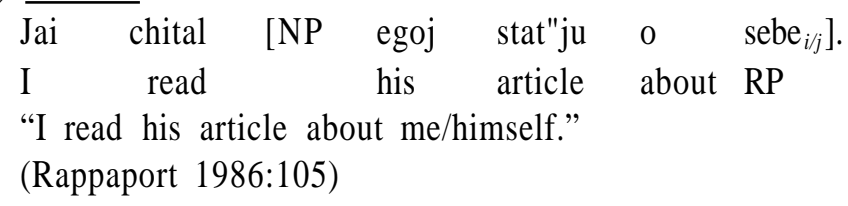

There are also languages (such as English) in which only a complex RP exists. In languages which have both a simplex and a complex RP, it is not uncommon for the complex RP to be a form derived from combining the simplex form and a corresponding personal pronoun:

(12) Chinese

$\begin{array}{llllll}\text { Zhangsan }_{i} & \begin{array}{l}\text { renwei } \\ \text { Zhangsan }\end{array} & \text { Lisi }_{j} & \text { zhidao } & \text { Wangwu }_{k} & \text { xihuan } \\ & & \text { Lisi } & \text { know } & \text { Wangwu like } & \\ \text { ta } & {\text { ziji } *_{* / *} / k} . & & & \\ \text { him } & \text { RP } & & & \end{array}$

"Zhangsan thinks Lisi knows Wangwu likes himself."

(Cole and Sung 1994:357)

The division between simplex and complex RPs, which can be traced back to Faltz (1985, but see also Pica 1987, 1991), is seen as very important as it has been found to be implicationally related to a number of other properties of RPs, such as presence/absence of phi-features (person, number, gender), being long-distance (e.g., the ability to refer to a clause-external antecedent), and subject-orientation (e.g., being restricted to reference to clausal subjects only, and not to objects). These are discussed in section 2.3. 


\subsection{Function and Meaning of RPs}

Traditionally, reflexivity is regarded as indicating a verbal activity which is directed back to the entity (usually the clausal subject) that initiated it. From a different perspective, reflexivity involves a predicate-argument structure (usually a clause, but sometimes also a noun phrase) in which two of the arguments have the same referent:

(13) [s He described himself as an honest man]

(14) $\left[{ }_{n p}\right.$ His description of himself $]$

Thus, the principal function of the RP is to impose a coreferential reading on the two arguments (while a non-RP in the same context wouldnormally be interpreted as disjoint in reference from the subject). This can be accomplished with a RP (as in the sentence above), with a clitic (e.g., in the Romance languages), or with a bound reflexive morpheme (e.g., in Russian).

The RP has also developed a number of related functions. It can be used to indicate an activity which, while not exactly directed back to the performing entity, is seen as strictly associated with this entity (sometimes referred to as 'middle voice'):

(15) Dutch

Oscar gedraagt zich.

"Oscar behaves himself." (Reuland 2001:451)

In some languages (e.g., English) it is often possible to omit the RP in such cases:

(16) Oscar behaved/washed/dressed (himself).

In addition, the RP can also be used to indicate passive meaning 
106 On the Form and Function of Reflexives in Artificial Languages

(as a rule, with inanimate subjects): there are languages (e.g., some of the Slavic languages) in which a reflexive clitic or a reflexive morpheme is used as an alternative device to form the passive voice:

(17) Bulgarian

\begin{tabular}{|c|c|c|c|}
\hline $\begin{array}{l}\text { a. Problem-at } \\
\text { problem-the }\end{array}$ & $\begin{array}{l}\text { beshe } \\
\text { was }\end{array}$ & $\begin{array}{l}\text { reshe-n } \\
\text { resolved-PASS }\end{array}$ & PARTIC \\
\hline $\begin{array}{l}\text { bord-a } \\
\text { board-the }\end{array}$ & $\begin{array}{l}\text { na } \\
\text { of }\end{array}$ & $\begin{array}{l}\text { direktori-te. } \\
\text { directors-the }\end{array}$ & \\
\hline $\begin{array}{l}\text { b. Problem-at } \\
\text { problem-the }\end{array}$ & $\begin{array}{l}\mathrm{se} \\
\mathrm{RP}\left({ }_{\mathrm{CL}}\right)\end{array}$ & $\begin{array}{l}\text { resh-i } \\
\text { resolve-PAST }\end{array}$ & $\begin{array}{l}\text { ot } \\
\text { from }\end{array}$ \\
\hline $\begin{array}{l}\text { bord-a } \\
\text { board-the }\end{array}$ & & $\begin{array}{l}\text { direktori-te. } \\
\text { directors-the }\end{array}$ & \\
\hline
\end{tabular}

Likewise, in some languages RPs can be used in impersonal constructions:

(18) Bulgarian

Vliza se prez goljama gradina.

enter $\quad \mathrm{RP}_{(\mathrm{CL})}$ through big garden

"You (can) enter (the house) through a large garden."

It is notunusual for a RP to be used to indicate reciprocal meaning, even if there is a distinct reciprocal pronoun:

(19) Bulgarian

Te se poglednaha.

they $\quad \mathrm{RP}_{(\mathrm{CL})}$ looked

"They looked at each other." 
From a discourse perspective, it seems that the principal function of the RP is to disambiguate between potential antecedents. Dowty (1980:32) has proposed that the distribution of reflexive and non-reflexive pronouns is regulated by the following principle:

(20) Neo-Gricean Conversational Principle

If a language has two (equally simple) types of syntactic structures $\mathrm{A}$ and $\mathrm{B}$, such that $\mathrm{A}$ is ambiguous between meanings $\mathrm{X}$ and $\mathrm{Y}$ while $\mathrm{B}$ has only meaning $\mathrm{X}$, speakers of the language should reserve structure $A$ for communicating meaning $\mathrm{Y}$ (since $\mathrm{B}$ would have been available for communicating $X$ unambiguously and would have been chosen if $\mathrm{X}$ is what was intended).'

This operates to eliminate ambiguity in sentences like (21):

(21) a. ${ }^{*}$ She $_{i}$ saw her . $_{\text {. }}$

b. She ${ }_{i}$ saw herself ${ }_{i}$. (Dowty 1980:32)

In the terms of the conversational principle formulated in (20), which we can informally call avoid ambiguity, the RP herself is the unambiguoussyntactic structure B: herself can refer only to the subject she and, therefore, is always chosen when such is the intended reference. On the other hand, the pronominal her can have a number of referents including she; her is, then, the ambiguous syntactic structure A which, in line with (20), is reserved for the cases when the intended reference is outside of the sentence.

While the proposed conversational principle captures what appears tobethebasicdiscoursefunction of RPs, it is clearly unable to account for all aspects of the distribution of anaphors, because the pattern we see in (21), and which is presumably the effect of Avoid ambiguity, can

\footnotetext{
${ }^{11}$ For a much more elaborated proposal, see Levinson (1991).
} 
also be observed in (22):

(22) a. *I saw me.

b. I saw myself.

Here, the pronoun me is as unambiguous as the RP myself and, therefore, should not be affected by the operation of avoid ambiguity. The opposite problem is manifested in sentences like (23) (note that judgements about such sentences seem to vary):

(23) a. $\operatorname{Max}_{i}$ saw a gun near himself $/$ him $i$.

b. Max $_{i}$ likes [NP jokes about himself ${ }_{i} /$ him $_{i}$ ].

(Reinhart and Reuland 1993:661)

In these examples, the pronoun him is ambiguous in that itcan refer not only to the nominal Max, but also to a clause-external entity. Thus, (23) represents exactly the case in which the proposed Avold AMBIGUiTY principle should apply, but it doesn't.

In fact, the neat pattern that is observed in sentences such as (21) and (22) is not as common as one might think, and the breakdown in the complementarity between reflexives and pronouns seems to be quite wide-spread. In the first place, there are languages (e.g., the Romance languages, but also some Germanic languages) in which there is no opposition between a reflexive and a non-reflexive form in the 1st and 2nd person altogether: the reflexive is only used in the 3rd person, while in the 1 st and 2 nd person non-reflexive personal pronouns are used:

(24) $\underline{\text { German }}$

a. Ich $_{i}$ hab' $\operatorname{mir}_{i}$ ein Auto gekauft.

I have me a car bought

"I have bought myself a car." 
b. $\operatorname{Er}_{i}$ hat $\operatorname{sich}_{i}$ ein Auto gekauft.

$\mathrm{He}$ has RP a car bought

"He has bought himself a car."

It is interesting to note that in languages in which there is a distinct reflexive form in the 1 st and 2 nd person, its use is often, but not always, optional and then it can freely be interchanged with a personal pronoun:

(25) Bulgarian

\begin{tabular}{|c|c|c|c|c|}
\hline $\mathrm{Az}$ & ne & mislja & $\mathrm{za}$ & mene/seb \\
\hline I & not & think & about & $R P$ \\
\hline
\end{tabular}

The same is not true for 3rd person sentences:

(26) $\operatorname{Ivan}_{i}$ ne misli za *nego ${ }_{i} /$ sebe $\mathrm{si}_{i}$. Ivan not thinks about him RP

"Ivan is not thinking of himself."

It should be noted that sentences like (23) and (25), in which there is a breakdown in the complementarity between reflexives and pronouns, are equally problematic for structural accounts of anaphora. It has been suggested (Farmer \& Harnish 1987; Reinhart \& Reuland $1991,1993)$ that the complementarity relation holds in cases in which the anaphor is a co-argument of the antecedent, as in (21) and (22), and breaks down in cases in which the anaphor is an adjunct, as in (23a), or is embedded in an argument, as in (23b). As a descriptive generalization, this seems to be fairly, but not completely, accurate as it fails to capture the fact that complementarity is observed for some anaphors which are prepositional arguments:

(27) I am mad at myself/*me. 
110 On the Form and Function of Reflexives in Artificial Languages

but nor for others:

(28) I am (not) thinking of myself/me.

The picture is additionally complicated by instances of logophoricity (see e.g., Sells 1987, Reinhart \& Reuland 1991) in which the use of the RP is arguably determined by "the subjective perspective of the referent of the antecedent of the reflexive" (Levinson 1991:122). Consider the following examples (from Reinhart \& Reuland 1991:311-2):

(29) a. She gave both Brenda and myself and a dirty look.

b. Lucie said that (you agreed that) a picture of herself would be nice on that wall.

c. Lucie boasted that the chairman invited her husband and herself for a drink.

d. She wrote that Max and herself are having a great time in Lima.

In the four sentences above, the use of the RP seems highly irregular as it is not in the same clause as its antecedent and represents a case of long-distance anaphora (see the following section), which is generally not allowed in English. Also, as observed in Reinhart \& Reuland (1991:313), logophoricity only seems to affect RPs which are either adjuncts, or are embedded in an argument. Analogous sentences in which the RP is actually an argument are ungrammatical:

(30) *Lucie boasted that the chairman invited herself for a drink. (Reinhart \& Reuland 1991:312)

It should be noted that languages seem to vary with regard to the logophoric use of RPs: empirical data suggest that such a use is rare or non-existent in some languages (e.g., Bulgarian). 
Finally, in some (but not all) languages, the RP is also used as an emphatic pronoun:

(31) The president himself will inspect the troops.

Many (e.g., König \& Siemund 1999) have observed that this fact is unlikely to be a coincidence, especially as it is found in a number of languages, not just a single language. There have been proposals (e.g., Faltz 1985) that historically the reflexive form has evolved from combining a pronoun and an emphatic element. Consider the situation in Old English, where no RPs existed and personal pronouns were used instead:

(32) pæt ic anigra me weana ne wende. that I any me hope not expected "that I expected no hope for myself."

(Beowulf 932-93, cited in van Gelderen 1999:191)

The form sylfne was originally used for emphasis:

(33) Sittan læte ic hine wið me sylf-ne. remain let I him with me self-ACC.M.SC "I let him remain with myself."

(Genesis 438, Junius Manuscript, cited in van Gelderen 1999:192)

However, it gradually became attached to the personal pronoun and came to mark coreference, not (only) emphasis.

\subsection{Phi-features, Long-Distance Anaphora, and Subject-Orientation}


112 On the Form and Function of Reflexives in Artificial Languages

There appears to be an implicational universal according which if simplex (monomorphemic) RPs are un- or underspecified for phi-features (person, nember, gender), they are defined as long-distance (LD), because they can take a clause-external antecedent, and they are subject-oriented, because they invariably select a clausal or a nominal subject as their antecedent. Consider the Icelandic RP sig. It is unspecified for person, number, or gender, and it can refer to a 'distant' antecedent, and it cannot have an object as its antecedent:

(34) Icelandic

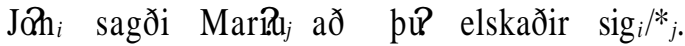

John told Maria that you loved RP

"John told Maria that you loved him."

(Cole and Sung 1994:359)

As the indexation in the example shows, the RP sig can only refer to the 'distant' matrix clause subject Jon, but not to the object Maria. The subject-orientation of simplex RPs is even more obvious within a single clause:

(35) Norwegian

$\mathrm{John}_{i}$ bad Maritj kikk-e bak $\mathrm{seg}_{j} /{ }^{*}$.

John asked Mary look-INFIN behind RP

"John ${ }_{i}$ asked Mary to look behind him $_{i}$."

(Hestvik 1992:578)

In contrast, complex RPs are generally specified for phi-features, they are not normally LD (e.g., they cannot take a clause external antecedent) and there are no restrictions on the class of potential antecedents they can select. A typical example of a complex RP is the English himself: it is completely specified for phi-features, and normally it can only refer to a nominal expression within the same clause: 
(36) a. $\mathrm{John}_{i}$ despises himself $\mathrm{i}_{\text {. }}$

b. ${ }^{*} \mathrm{John}_{i}$ thinks that Mary despises himself ${ }_{i}$.

and it is not subject-oriented (e.g., it can select either the subject or the object as its antecedent):

(37) $\mathrm{John}_{i}$ told Bill $l_{j}$ about himself $f_{i} /_{\text {. }}$

It shouldbenotedthat, whileRPsinmostlanguagesseem to follow this pattern, there are nevertheless a number of exceptions suggesting that it might be more correct to see this as a strong crosslinguistic tendency rather than as a true universal. For instance, the forms of complex RPs in a number of Germanic languages (e.g., the Dutch zichself, the Norwegian seg selv, the Icelandic sjalfur sig) are as underspecified for phi-features as the respective simplex RPs (zich,seg, sig): both types are only marked for (3rd) person. On data provided in Toman (1991), the Czech RP is a featureless monomorphemic reflexive, but contrary to expectations it behaves like a complex, rather than simplex, RP: it is neither LD, nor subject-oriented:

(38) $\mathrm{Karel}_{i}$ narovnal desticky ${ }_{j}$ na sebe $_{i} /$. $_{\text {. }}$ Karl stacked plates on RP

"Karl stacked the plates on himself/one another." (Toman 1991:155)

The Bulgarian RP sebe si also breaks away from the general pattern: it clearly is a complex RP consisting of the bound reflexive morpheme sebe 'self' (which, similarly to the English self, is also a word-formative prefix) and the reflexive possessive clitic si, but it is completely void of phi-features and is subject-oriented. 
114 On the Form and Function of Reflexives in Artificial Languages

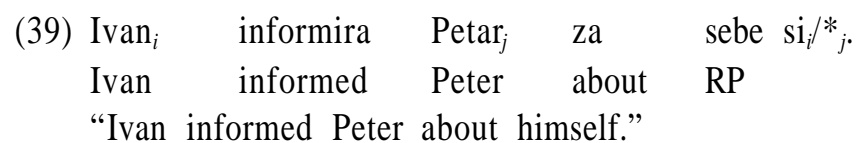

\subsection{Subject RPs and RPs inside the Subject}

Studies investigating RPs report that in some languages they are barred from occurring in subject position (which in the following examples is marked with square brackets):

(40) *John said that [himself] would come.

while in others there is no such restriction:

(41) Chinese

$\begin{array}{lllll}\text { Zhangsan }_{i} & \text { shuo } & {\left[\text { ziji }_{i}\right]} & \text { hui } & \text { kai. } \\ \text { Zhangsan } & \text { say } & \text { RP } & \text { will } & \text { come }\end{array}$

"Zhangsan said that he would come."

(Progovac 1993:759)

As this is an issue that bears on the distribution of RPs, it will briefly be considered here. (41) indicates that in principle RPs can occur in subject position. The question that needs to be addressed then is why RPs in English (and a number of other languages) cannot. In the view taken here, there are at least two relevant reasons why himself is ruled out in sentences like (40): firstly, himself as a non-long-distant RP needs to take an antecedent in the same clause, but if it is in subject position, there would be nothing hierarchically higher that can actually function as its antecedent (see footnote 6); secondly, it is quite common for RPs to be marked for objective case, and consequently in languages which mark the subject for the nominative, RPs would be barred from occurring in subject position, because they are marked for a different case. This, of course, should not bar them from occurring 
inside the subject:

(42) a. [John's description of himself] surprised us.

b. [The way John described himself] surprised us.

This completes our (necessarily brief) overview of the distribution and use of RPs in natural languages. In the next part of our paper, we shall review RPs in a number of artificial languages.

\section{Artificial Languages}

Our survey of RPs in ALs is somewhat random, being restricted to ALs about which we have a fair amount of information, and indeed, not even all of those are discussed here. Adequate information may not be easy to come by, as primary works are often old and not widely distributed. Given such limitations, weh opet ogives omeide a of what has been done by language designers in this area of the grammar.

Most artificial languages are based largely on natural languages, and so it is not surprising if their reflexive words resemble or are identical to reflexive words in natural languages. As noted above, there apparently are natural languages without RPs, their function being fulfilled by middle orreflexive verb forms or in some other way. There seem to be few, if any, ALs which express reflexivity only through verbal inflection. Volapük has a verbal affix which marks reflexivity (and which can appear in more than one position relative to person and number affixes). In the singular one has the option of using a separate RP instead of the verbal marker. ${ }^{12}$ Inthe3rdperson,thisRP is identical to the affix, except that it bears a case marker; in other persons it is identical to the personal pronouns:

\footnotetext{
${ }^{12}$ In the plural the use of separate pronouns is used for a reciprocal reading (only), which is why reflexivity must be signaled by the verbal affix.
} 
116 On the Form and Function of Reflexives in Artificial Languages

(44) a. Vatük-ob.

wash-1SG

"I wash."

b. Vatük-ob-ok.

wash-1SG-REFL

"I wash myself."

c. Vatük-ob ob-i.

wash-1SG I-ACC

"I wash myself." (Sprague 1888)

The following example from the AL American is intriguing:

(45) $\$ 8$ agi maxtom facir $\$$. $0-\mathrm{m}$

it has power make-INFIN it-ACC

mor-ermoso-m $\cdots$

more-beautiful-ACC

"...-it has the power to make itself more beautiful."

(O'Connor 1917:38)

Here a 3rd person personal pronoun is being used as a RP. It is not clear to us whether this was the general practice which O' Connor intended to prescribe: if so, American would be like Gumbaynggir in not (obligatorily) distinguishing personal and reflexive pronouns. One question, perhaps especially relevant to a priori languages, is whether RPs are etymologically related to any other type of word; this is roughly equivalent to the question of whether RPs are monomorphemic or polymorphemic. To our knowledge there are no artificial languages with RPs of the type illustrated by Kabuverdiano, Sanuma, Finnish, or Zande.

Some a priori AL have lexical systems in which lexemes with similar meanings or of similar types share one or more sounds in 
common. One could argue that in such systems there are no monomorphemic words, and so RPs could be seen as polymorphemic, but not in the way considered by those making this distinction among reflexives. For example, the $\mathrm{Oz} \mathrm{RP}$ is $e k$, of which the $e$ - seems to be an element shared by other pronouns, e.g., ep 'I, me'. The possessive form is $k e k$.

Some ALs have RPs which are monomorphemic and have no phi-features, e.g., Unitario's sin. However, the RPs of the majority of ALs, whether monomorphemic or polymorphemic, seem to have at least one phi-feature. The RP of Veltparl, evidently borrowed from English, is monomorphemic and has no phi-feature for person, as shown by the following:

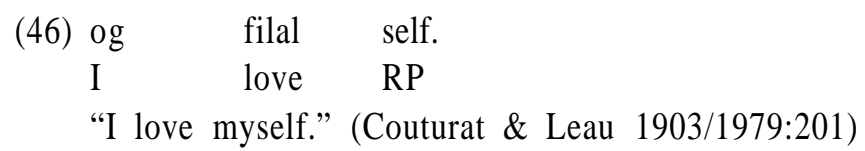

It does apparently have the number phi-feature, since the plural form is selfy.

Some ALs have RPs which are monomorphemic and have phi-features for person. There are two ways in which this can happen: 1) In many languages, there is only a distinct RP for 3rd person; in the other persons the personal pronoun is also used as a RP. Therefore, if the distinct RP is used, it is 3rd person, by default, one might say. 2) In other languages, all RPs are distinct from personal pronouns in all persons, and there are distinct RPs for all persons.

The former case holds in Esperanto, whose (3rd person) RP is $s i$, for both singular and plural, and all genders; it, therefore, has the phi-feature for person, but not for gender or number. The same is true of Interlingua (IALA) and Romanova, both of which have se as their (3rd person) RP.

Other ALs have polymorphemic RPs, all of which seem to involve a personal pronoun, or an associated possessive form and a morpheme 
meaning 'self'. These include several ALs based on English, or partly on English, such as Anglo-Franca. In this language, there are no case forms of personal pronouns, e.g., the nominative is the only form, and the RPs also contain this form, e.g., theyselfs. Note that plurality is marked on both parts of this, e.g., the personal pronoun and the 'self' part. $^{13}$ The RPs of Olingo consist of possessive forms and -ego 'self', e.g., hiaego "himself."

The general RP of Eurolengo is sel, for all persons and numbers. It is thus monomorphemic and lacks phi-features; as Jones (1972:5) says, "Add the word 'sel' at all stages." However, he uses the form lasel for 'herself'; that is, for the feminine singular 3rd person RP, and apparently only for it, there is a polymorphemic form with phi-featurs:

(47) Entretempo my sposa musto-preparar le

Meanwhile my wife must prepare the

$\begin{array}{lllll}\text { lunch for lasel } & \text { and } & \text { le } & \text { kinders } \\ \text { lunch for herself } & \text { and } & \text { the children (ibid.:24) }\end{array}$

Let us now turn to the use of RPs in artificial languages. Information on this is harder to gather than information about forms,

\footnotetext{
${ }^{13}$ Unitario has the curious feature that what would appear from their form to be reflexive pronouns areusedasnon-subjectpronouns(andnotasRPs,asfar as we can tell):

(i) ilo se-mismo tenas.

he/she/it him/her/it holds

"She holds it." (Pleyer 1990:27; OT)

There are other non-subject pronouns, such as $l o$ 'him/her/it', but the use of the -mimso formsdoes notseem tobeaquestionofemphasis, oratleastnotonlythat. As noted above, the RP of thislanguage is sin.

${ }^{14}$ Given theexistence of lasel, it maybemoreaccuratetosay that seldoeshavesome phi-features, namely [+MALE,-PLURAL], than to say it has no features.
} 
because authors often give less information about syntax than about morphology. In addition, whether or not explicit information is given, one may want to check texts in the language for syntactic data, but often there is not enough textual material in a language to fully determine some points of syntax. Onemightwanttobearinmindthat RPs have (or should have) a pragmatic function, the disambiguation of possible readings, and a syntactic/semantic function, imposing co-referential readings on two noun phrases.

We know of no instances of RPs in ALs being used in constructions which are clearly passive, e.g., which have an expressed agent, as in the natural language example (17b). However, in some ALs, RPs can be used in so-called 'middle' constructions. This is explicitly statedwith respect to Interlingua (IALA): "Reflexive constructions are also used to express passive ideas when there is no agentinvolved. 'These books are sold at Bloomingdale' s' may be replaced by the translation of 'These books sell themselves at Bloomingdale's'. Note that this covers constructions of the type, "These books sell well." (Gode and Blair 1951:25). One of their examples is:

(48) Tal cappellos se vide frequentemente. such hats RP see frequently "Such hats are often seen" or "One often sees such hats." (ibid.)

Eurolengo's RP is also used in this way:

(49) Pipes fumo sel mor in le Unitado Kingdom... Pipes smoke RP more in the United Kingdom "Pipes are smoked more in the United Kingdom..." (Jones 1972:46)

However, in the same passage, and with the same verb, we find a true passive construction: 
120 On the Form and Function of Reflexives in Artificial Languages

$$
\begin{aligned}
& \text { (50) so mucho as ever ist-o fum-ado. } \\
& \text { as many as ever be-PRES smoke-PAST PARTIC } \\
& \text { "as many as ever are smoked" (ibid.) }
\end{aligned}
$$

It is unclear to us why constructions have been chosen here or whether they are in free variation. To our knowledge, no AL has an impersonal use of RPs, as Bulgarian does.

There seem to be few, if any, ALs in which RPs are freely used as reciprocal pronouns.

Like natural languages, ALs vary with respect to identity of form of RPs and intensive pronouns. For example, Esperanto has a separate form for the latter, mem. The same seems to be true of INTAL, in which self is found as an intensive pronoun (as opposed to se, the 3rd person RP). On the other hand, Olingo uses RPs as intensives, e.g.,:

$$
\begin{aligned}
& \text { (51) Imiaego uri bonfortuna. } \\
& \text { Imyself am good.fortune } \\
& \text { "I myself am good fortune." (Jaque 1944:29) }
\end{aligned}
$$

In Eurolengo and aUI as well there seems to be identity of form of RPs and intensive pronouns. In the Blue Language the situation is not clear, at least as presented in our source on this language, Bollack (1900). On p. $20 s u$ is said to be a RP, not declined for person, and on the same page a series of "emphatic" pronouns is given, which do have phi-features, e.g., eme 'myself', and ete 'thyself'. However, on p.57 is the following sentence in which the emphatic pronoun seems to be functioning as a RP:
(52) Et keni ete!
thou(VOC) know ete
"Know thyself!" 
We cannot determine whether this is simply an error on the part of the author, or whether he meant to allow for such usage of the "emphatic" pronouns.

Pantos-Dimou-Glossa apparently has an unusual type of identity of form, between RPs and indefinite pronouns.

As far as we know, no language designer explicitly allows for logophoric use of RPs. This may not be surprising, considering that logophoricity has only come to the attention of even theoretical linguists in the last two decades, and is something which native speakers and language designers may not be consciously aware of. However, it also would not be surprising if in actual use, RPs were sometimes used as logophoric pronouns.

Very few sources on ALs discuss whether RPs can be long-distance. The detailed grammar of Esperanto Wennergren (2001) is explicit on this point: generally Esperanto does not allow LD reflexives. This is shown by the following pair of sentences:

(53) a. Li ordon-is al la servisto.

$\mathrm{He}$ order-PAST to the servant

vest-i si-n.

dress-INFIN RP-ACC

"He ${ }_{i}$ ordered the servant ${ }_{j}$ to dress himself* ${ }_{i j /}$ "

b. Li ordon-is al la servisto

$\mathrm{He}$ order-PAST to the servant

vest-i li-n.

dress-INFIN he-ACC

"He $\mathrm{H}_{i}$ ordered the servant ${ }_{j}$ to $\operatorname{dress}_{\operatorname{him}_{i}} *_{j .}$."

(Wells 1969:21)

Note that this is contrary to the implicational universal for natural 
languages mentioned above. Given that $s i$ is monomorphemic and is not fully specified for phi-features, one would expect it to be long-distance. There are some circumstances in which it can be long-distance, for example in an infinitive phrase with no overt subject:

(54) La rego send-is vok-i si-a-n the king send-PAST call-INFIN RP-ADJ-ACC

kuracisto-n. doctor-ACC

"The king ${ }_{i}$ sent (someone) to call his ${ }_{i}$ doctor."(PMEG; OT)

The Esperanto RP is subject-oriented, and is explicitly said (e.g., in PMEG) to be unable to act as a subject. Unfortunately, detailed and clear information is usually lacking in descriptions of other ALs. Consider the remark by Gode and Blair (1951:25), which is, to our knowledge, one of the most detailed sources for the grammar of Interlingua (IALA): "Reflexive pronouns are primarily of the type in which the object of the verb happens to be logically identical with the subject." The question is what is meant by "primarily": does it allow for non-subject-orientation, or for the RP to be something other than a verbal object (e.g., the object of a preposition), or both?

$\mathrm{Oz}$ is the only AL that we know of in which an RP can act as a subject (and indeed as the subject of a finite clause):

(55) ap ipOv ed ek pinfoid at.

he said that RP loved her

"He $e_{i}$ said that he ${ }_{i}$ loved her." (Elam 1932:26)

Elam (ibid.:16) describes $e k$ as "referring only to the subject of the proposition in which it is used". Thismeansthatitissubject-oriented, but given examples like the one above, we might not interpret Elam's words to mean it is not long-distance, e.g., "proposition" maynotmean 
"clause".

$\mathrm{Oz}$ is unusual for another reason: the RP can refer to an antecedent which is not in a hierarchically higher structural position and does not c-command it (if the following sentence does not contain an error):

(56) hEv az ansAlUt iftlEplezais k-ek because the wicked not.immediately.receive RP-POSS

anpAtpyaup ek iftEgtOg adpad astlaup.

just.deserts RP grow.bold on transgression

"Because the wicked $_{i}$ do not receive their ${ }_{i}$ just deserts immediately, they $y_{i}$ grow bold in transgression." (ibid.:36)

Here, az ans AlUt does not c-command $e k$ (because it is in a subordinate clause), and yet it functions as the antecedent for the RP.

The conditions under which the (3rd person) possessive reflexive and RP of Hom-idyomo (sua and se respectively) are supposed to be used are unusual. Cárdenas (1923:33) says of the former:

The use of some of the third-person possessives may give rise to ambiguity. In order to avoid it, use is made of the substitute possessive sua, which governs the complement of a sentence or clause having two subjects, provided (1) the two subjects are of the third person; (2) they have the same number and gender; (3) the complement refers only to the first of the two subjects.

In other words, the reflexive possessive should only be used to indicate coreference to the first of two conjoined 3rd person subjects which have the same phi-features, as in the following example:

\footnotetext{
${ }^{15}$ Elam (1932) doesnotgive enough information forustobeabletodividesome of the words hereintotheirconstituentmorphemes,but this is not ofsignificance for the issue at hand.
} 
124 On the Form and Function of Reflexives in Artificial Languages

(57) Mary kay Kate irey a sua domo.

Mary and Kate went to sua house

"Mary $i$ and Kate went to her ${ }_{i}$ house." (ibid.)

Cárdenas (ibid.:34) says that "The same rules apply to the reflexive se." However, in the following sentence, he uses se when the above conditions do not hold, namely when there is a non-conjoined subject:

(58) La marito adresey se a una tansport-agentio.... the husband betook RP to a transportation-agency

"The husband betook himself $_{i}$ to a transportation agency...."

As far as we know, no natural language has distinct subject- and object-oriented RPs. The logic-based AL Lojban has something close to them; however, it has a series of RPs which are used to indicate coreference with different arguments of the predicate, e.g., vo'a for coreference with the first argument, vo'e with the second argument, and so on. Below is an example of one of these in use:

$$
\begin{aligned}
& \text { (59) mi klama le } \operatorname{zarc}_{i} \quad v o^{\prime} e . \\
& \text { I go the store RP } \\
& \text { "I go to the store from itself }{ }_{i} \text { " } \\
& \text { (Lojban Reference Grammar chapter 7.8) }
\end{aligned}
$$

The RP vo'e is used, since coreference with the second argument of klama is intended. We could, therefore, call it a 2nd argument-oriented RP (which is not the same as object-orientation; the 2nd argument in Lojban does not always correspond to the traditional

\footnotetext{
${ }^{16}$ Weareusing thesymbol $<\mathrm{r}>$ foraletter ofHom-idyomowhichwecannotproduce here;it resemblesa script letter $\left\langle\mathrm{r}>\right.$ andis"pronouncedlikeSpanishandItalian $r^{\prime \prime}$ (Cárdenas ibid.:3)
} 
notion of object), while $v o^{\prime} a$ is a 1 st argument-oriented RP. This system seems rather different from what happens in natural languages.

Having seen RPs and their functions in a range of ALs, we shall now give our opinions on how they should be formed and used.

\section{Recommendations}

We shall first make two general recommendations, e.g., recommendations not restricted to RPs. It sometimes happens that designers of artificial languages do not seem to follow their own rules, e.g., there is occasionally a mismatch between the rule that they propose and the practice that they follow in the texts which they provide. We have seen a possible instance of this with the Blue Language. This can make it difficult to describe or analyze the language, and perhaps more importantly, could create problems for learners.

Therefore, language designers should take care not to make errors in the descriptions of their languages, and in the texts they write in them. Also, designers should be very explicit about the properties of the language. For example, with respect to RPs, they should state whether they are subject-oriented, long-distance, and so on.

As with other aspects of consciously designed languages, there is a potential conflict between clarity and simplicity. One could have an AL (or part of one) which is very simple, but in which ambiguity or unclarity will frequently arise, or a language in which such problems very rarely occur, because there is a complex system for that part of the grammar. American may represent the greatest simplicity in the domain of RPs, it does not seem to have any, and personal pronouns can be used reflexively, even in the 3rd person. There is, however, a cost of ambiguity in the 3rd person, e.g., (45) apparently can also mean "it $t_{i}$ has the power to make $i_{i}$ more beautiful". This raises the question 
of how much ambiguity is acceptable for an AL, and what types of ambiguity are acceptable. One might feel that the kind of ambiguity that arises in American is unacceptable, although it may be difficult to provide a principled basis for what is an unacceptable degree or type of ambiguity. However, the fact that there are almost no natural languages which are ambiguous in this way to a large extent might be taken as support for the position that an optimal AL should not allow it, and hence should have some overt means for disambiguation.

As pointed out at the beginning of this paper, pronouns are referentially deficient and therefore are intrinsically ambiguous. If the designer's goal is to avoid ambiguity at all costs, one (rather extreme) way to do it would be to eliminate both reflexive and personal pronouns and simply repeat noun phrases, e.g., John saw John. This would eliminate much ambiguity, but very few, if any, ALs have done this. One might ask why not. One possible reason is that it is inefficient, especially in the case of long noun phrases. Further, it may be aesthetically displeasing, though the role which such factors should have is not clear to us.

While we recognize that striking a good balance between clarity and simplicity is essential in designing a grammatical form with a specific function (as is the case with RPs), we believe that it is also very important to consider the construction of an $\mathrm{AL}$ from a different perspective: viz. the perspective of the language learner and language user. In terms of language acquisition, ALs are by their nature non-primary languages; in other words, an AL is almost always learned and used as a second language. This being the case, the view taken here is that the specific properties of second language acquisition must be taken into account in the construction of an AL. This is clearly a huge issue whose discussion is well beyond the goals of this paper and which certainly deserves separate treatment.

As regards the form of the RP in ALs from the perspective of their prospective users, in second language acquisition literature there is now substantial evidence thatin the acquisition of the grammaticalstructures 
of a second language, learners display a distinct preference for form-function relationships which are both transparent and isomorphic. What this means is that second language learners are more successful in acquiring grammatical structures whose function issufficiently clear, and tend to establish a one-to-one relationship between a grammatical form and its function. The implications for the form of RPs in ALs are obvious: the RP must have a distinct form, it must be as simple as possible, and it must be associated with a distinct single function.

As we have noted, some natural and artificial languages indicate reflexivity through verbal inflection (e.g., the middle voice forms of Classical Greek). A language with such verb forms would not need reflexive accusative pronouns, but it might be thought good to have pronominal forms for reflexive indirect objects and/or objects of prepositions. Wewouldarguethat thisisnotanefficientsystem--if one is going to have RPs, it would be better to have them for the whole range of possible non-subject functions, rather than adding verb forms which will complicate the verbal paradigms.

If an $\mathrm{AL}$ designer opts for a reflexive form which is a pronoun, from the perspective of the AL learner and user it would appear that that a reflexive form based on, or derived from, the personal pronoun system of that AL would be easiest to learn and use: in such a case, the learner will only need to learn the form of a simple reflexive morpheme (e.g., se or si) and that it is attached to the respective form of the personal pronoun to form a RP (e.g., me-se or se-me).

There are many languages, both artificial and natural,which do not have distinct RPs for the first and second persons. This does not lead to ambiguity, as it would in the 3rd person; however, one could argue that having 1st and 2nd person RPs adds a level of clarity (desirable for the second language learner) and redundancy. For example, if one does not hear the subject of a sentence, when myself is the object, the subject can be reconstructed to be $I$. However, this greater redundancy comes at the cost of having a slightly more complex system of pronouns to be learned. 
Another question is whether RPs should have overt marking for phi-features, and if so, for which ones. Again, we feel that having at least person phi-features would be desirable from the point of view of the 2nd language learner. If the model for the formation of RPs suggested above is adopted, then they will inherit the phi-features of the pronoun from which they are formed.

As regards the function of RPs in ALs, we would recommend that they be used for a single purpose, viz. to mark reflexivity. We, therefore, think that an optimal AL would have separate forms for reciprocal and reflexive pronouns. Again this involves a question of whether a certain kind of ambiguity is acceptable, or whether it is worth adding a form to the grammar to eliminate it. Given the 2nd language orientation of ALs and 2nd language learners' preference for isomorphic form-function relations, we feel it is better to opt for clarity. The same remarks apply to intensive pronouns. Although some languages, both natural and artificial, employ the same forms for RPs and intensives, we feel that an optimal AL would have distinct forms. Likewise we do not recommend having the same forms for RPs and indefinite pronouns, as Pantos-Dimou-Glossa does. We also suggest avoiding unusual rules for use of RPs, like those of Hom-idyomo.

We now turn to some more subtle questions of the function of RPs. First, let us consider whether RPs should be subject-oriented. Consider the following sentence:

(60) John told Bill about himself.

If the $\mathrm{RP}$ in an $\mathrm{AL}$ equivalent of this sentence were subject-oriented, then that sentence would not be ambiguous, unlike the English version. Given that subject-orientation of RPs is quite wide-spread crosslinguistically, and that speakers generally have no problems identifying the subject as a syntactic constituent, it is unlikely that stipulating subject-orientation for RPs would significantly add to learnability or processability of ALs. There is another, possibly very 
important, aspect of the subject-orientation of RPs.Atthebeginning of the paper, we briefly referred to c-command as a hierarchical structural relation that generally holds between anaphors and their antecedents in natural languages. Why there should be such a complex configurational relation is a question that, to the best of our knowledge, no one has answered (or, for that matter, even attempted to answer). Considering its universality, however, and that it may be relevant to other components of grammar, c-command is likely to be a necessary condition for language-based communication systems, and therefore shouldnotbeexcluded from the specification of ALgrammars. In view of its complex nature, it would, however, pose huge learnability problems. The advantage of having subject-oriented RPs, apart form reducing potential ambiguity, is that by virtue of being the hierarchically highest constituent within the clause, the subject c-commands all other constituents. Thus, subject-orientation entails c-command without the need to explicitly learn it.

Let us consider whether long-distance RPsshould be allowed in an AL. Although they occur in some natural languages, weconsider them to be a complicating factor in a language designed for 2nd language learners. The simplest rule would be one which restricted possible antecedents of an RP to those in the same clause, whether it is finite or not, or to those in the same NP. A hearer (or reader) would then only need to search in that clause (orNP)forpossible antecedents, and would not need to consider potential antecedents which had occurred earlier, e.g., in a matrix clause containing the clause in question. We would favorsuch a rule,with noexceptions (unlike Esperanto); itcould lead to ambiguity in some cases, but such ambiguity would often be obviated by having subject-oriented RPs with phi-features, and we believe the remaining potential for ambiguity is outweighed by the simplicity resulting from forcing RPs to select their antecedents in the same clause or NP.

If there are no LD reflexives, and if we assume that an $\mathrm{AL}$ will have the same sorts of conditions on the relationbetween anaphors and 
130 On the Form and Function of Reflexives in Artificial Languages

their antecedents as natural languages (e.g., involving c-command), then RPs will never be able to be subjects.

Taking all the above into account, in our view the optimal RP would be a polymorphemic one, which bore phi-features, and which clearly was reflexive, which was distinct in form from both reciprocals and intensives, and which was subject-oriented, but not long-distance. Further, all of these facts should be clearly and explicitly stated in the designer's presentation of his language.

\section{References}

Bollack, L. 1900. Abridged Grammar of the Blue Language. ("English version by Professor Tischer"). Ms., Paris.

Borgman, D. M. 1990. Sanuma. In D. C. Derbyshire \& G. K. Pullum (eds.), Handbook of Amazonian Languages 2, 15-248.

Camenas, C. 1923. Hom-idyomo (second edition). Ms., Leipzig.

Chapman, S. \& D. C. Derbyshire. 1991. Paumar짜타므 D. C. Derbyshire \& G. K. Pullum (eds.), Handbook of Amazonian Languages 3, 161-352.

Chomsky, N. 1981. Lectures on Government and Binding. Dordrecht: Foris.

Chomsky, N. 1986. Knowledge of Language: Its Nature, Origin, and Use. New York: Praeger.

Chomsky, N. \& H. Lasnik. 1992. The Principles and Parameters Theory. In J. Jacobs, A.vonStechow, W. Sternefeld, \& T. Vennemann (eds.), Syntax: Ein Internationales Handbuch Zeitgen 双ischer Forschung 506-569.

Cole, P. \& L. Sung. 1994. Head Movement and Long-distance Reflexives. Linguistic Inquiry 25, 355-406.

Couturat, L. \& L. Leau. 1903, 1907/1979. Histoire de la Langue Universelle. Hildesheim: Georg Olms Verlag.

Dowty, D. R. 1980. Comments on the Paper by Bach \& Partee. In K. J. Kreiman \& A. E. Oteda (eds.), Papers from the Parasession on Pronouns and Anaphora 29-40.

Eades, D. 1979. Gumbaynggir. In R. M. W. Dixon \& B. Blake (eds.), Handbook of Australian Languages 1, 245-361.

Elam, C.M.1932. The CaseforanAPrioriLanguage. Cincinnati: The Open 
Sesame Press.

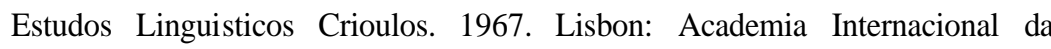
Cultura Portuguesa.

Faltz, L. M. 1985. Reflexivization: A Study in Universal Syntax. New York: Garland.

Farmer, A. \& M. Harnish. 1987. Communicative Reference with Pronouns. In M. Papi \& J. Verschueren (eds.), The Pragmatic Perspective 547-565.

Gode, A. \& H. E. Blair. 1951. Interlingua. New York: Storm Publishers.

Hestvik, A. 1992. LF Movement of Pronouns and Antisubject Orientation. Linguistic Inquiry 23, 557-594.

Jaque, R. S. 1944. One Language. Santa Barbara: J. F. Rowny Press.

Jones, L.1972. Eurolengo, the LanguageforEurope: A Practical Manualfor Business and Tourism. Newcastle upon Tyne: Oriel Press.

König, E. \& P. Siemund. 1999. Intensifiers and Reflexives: A Typological Perspective. In Z. Frajzyngier \& T. Curl (eds.), Reflexives: Forms and Functions 41-74.

Koster,J. \& E. J.Reuland. (eds.). 1991. Long-distance Anaphora. Cambridge: Cambridge University Press.

Lees, R. \& E. Klima. 1963. Rules for English Pronominalization. Language 39, 17-28.

Levinson, S. C. 1991. Pragmatic Reduction of the Binding Conditions Revisited. Journal of Linguistics 27, 107-161.

Lojban Reference Grammar. Available at URL <http://www.lojban.org/ publications/reference_grammar.html> (version current on February 19, 2002).

Manzini, R. \& K. Wexler. 1987. Parameters, Binding Theoryand Learnability. Linguistic Inquiry 18, 413-444.

Morneau, R. 1993. Designing an Artificial Language: Anaphora (revised version). Available at URL <http://www.srv.net/ ram/anaphora.html>. (version current on 9 November 2001).

Moskovsky, C. 2001. On Reflexive and Pronominal Binding in Bulgarian. Contrastive Linguistics 26.3, 33-63.

O'Connor, C. L. 1917. American: The New Pan-American Language. Ms., Buffalo.

Pica, P. 1987. On the Nature of the Reflexivization Cycle. Proceedings of NELS 17.2, 483-499. 
132 On the Form and Function of Reflexives in Artificial Languages

Pica, P. 1991. On the Interaction between Antecedent-government and Binding: The Case of Long-distance Reflexivization. In J. Koster \& E. Reuland (eds.), 119-136.

Pleyer, M. 1990. Unitario. Bensheim: Unitario Press.

Pollard, C. \& I. Sag. 1992. Anaphors in English and the Scope of the Binding Theory. Linguistic Inquiry 23, 261-303.

Progovac, L. 1993 Long-distance Reflexives: Movement-to-infl Versus Relativized Subject. Linguistic Inquiry 24, 755-772.

Rappaport, G. 1986. On Anaphor Binding in Russian. Natural Language and Linguistic Theory 4, 97-120.

Reinders-Machowska, E. 1991. Binding in Polish. In J. Koster \& E. Reuland (eds.), 137-150.

Reinhart, T. 1983. Anaphora and Semantic Interpretation. London: Croom Helm.

Reinhart, T. \& E. J. Reuland. 1991. Anaphors and Logophors: An Argument Structure Perspective. In J. Koster \& E. Reuland (eds.), 283-321.

Reinhart, T. \& E. J. Reuland. 1993. Reflexivity. Linguistic Inquiry 24, 657-720.

Reuland E. J. 2001. Primitives of Binding. Linguistic Inquiry 32, 439-492.

Schladt, M. 1999. The Typology and Grammaticalization of Reflexives. In Z. Frajzyngier \& T. Curl (eds.), Reflexives: Forms and Functions 103-125.

Sells, P. 1987. Aspects of Logophoricity. Linguistic Inquiry 18, 445-481.

Sprague,C.E.1888.HandbookofV olapük. The Office Company,NewYork. Available at URL <http://personal.southern.edu/ caviness/Volapuk/ HBoV/>. (version current on 9 November 2001).

Toman, J. 1991. Anaphors in Binary Trees: An Analysis of Czech Reflexives. In J. Koster \& E. Reuland (eds.), 151-170.

Tucker, A. N. \& M. A. Bryan. 1966. Linguistic Analyses: The Non-Bantu Languages of North-Eastern Africa. London: Oxford University Press.

van Gelderen, E. 1999. Bound Pronouns and Non-local Anaphors: The Case of Earlier English. In Z. Frajzyngier \& T. Curl (eds.), Reflexives: Forms and Functions 187-226.

Wells, J. C. 1969. Esperanto Dictionary. New York: David McKay.

Wennergren, B. 2001. PMEG: Plena Manlibro de Esperantiko Gramatiko. Available at URL <http://www.bertilow.com/pmeg/pemg10.index.php> (version currenton260ctober2001).(We refer to this work as PMEG) 
Zribi-Herz, A. 1989. Anaphor Binding and Narrative Point of View: English Reflexive Pronouns in Sentence and Discourse. Language 65, 695-727. 